\title{
Frequency response of the Leap Motion Controller and its suitability for measuring tremor
}

\author{
Clay J. Kincaid \\ Brigham Young University \\ Austin C. Vaterlaus \\ Brigham Young University \\ Nathan R. Stanford \\ Brigham Young University \\ Steven Knight Charles \\ skcharles@byu.edu
}

Follow this and additional works at: https://scholarsarchive.byu.edu/facpub

Part of the Mechanical Engineering Commons

\section{Original Publication Citation}

C. J. Kincaid, A. C. Vaterlaus, N. R. Stanford, and S. K. Charles, "Frequency response of the Leap Motion Controller and its suitability for measuring tremor," Medical Engineering \& Physics, In press.

\section{BYU ScholarsArchive Citation}

Kincaid, Clay J.; Vaterlaus, Austin C.; Stanford, Nathan R.; and Charles, Steven Knight, "Frequency response of the Leap Motion Controller and its suitability for measuring tremor" (2018). Faculty Publications. 2716.

https://scholarsarchive.byu.edu/facpub/2716

This Peer-Reviewed Article is brought to you for free and open access by BYU ScholarsArchive. It has been accepted for inclusion in Faculty Publications by an authorized administrator of BYU ScholarsArchive. For more information, please contact ellen_amatangelo@byu.edu. 


\title{
Frequency response of the Leap Motion Controller and its suitability for measuring tremor
}

\author{
Clay J. Kincaid ${ }^{1}$, Austin C. Vaterlaus ${ }^{1}$, Nathan R. Stanford ${ }^{2}$, Steven K. Charles ${ }^{1,2}$ \\ ${ }^{1}$ Mechanical Engineering and ${ }^{2}$ Neuroscience, Brigham Young University, Provo, UT 84602
}

\section{Disclosures}

Kincaid and Vaterlaus: none

Stanford and Charles: NR Stanford is CEO and Co-Founder of, and holds stock in, Vykon Technologies LLC. SK Charles is a scientific advisor to, and holds stock in, Vykon Technologies LLC. This company has licensed technology invented by NR Stanford and SK Charles to develop markerless monitoring of movement disorders such as monitoring of tremor using depth sensing cameras such as the Leap Motion Controller. 


\section{Abstract}

Although tremor is one of the most common movement disorders, it is evaluated using relatively coarse clinical scales. We propose to measure tremor in clinical settings using the Leap Motion Controller (LMC), which is a markerless motion capture sensor that has a low cost, zero set-up time, and dynamic accuracy of $1.2 \mathrm{~mm}$. However, the frequency response of the LMC has not been characterized, so its ability to track oscillations such as tremor is unknown. To characterize the frequency response of the $\mathrm{LMC}$, we measured the position of a mannequin hand simultaneously with the LMC and a high-resolution encoder while the mannequin hand oscillated at various combinations of frequency $(1-15 \mathrm{~Hz})$ and amplitudes $(0.01-30 \mathrm{~mm})$. We calculated the magnitude ratio and phase shift of the LMC and found the bandwidth of the LMC to range from $0-3 \mathrm{~Hz}$ to 0 $5 \mathrm{~Hz}$ for tremor amplitudes greater than the dynamic accuracy. This bandwidth is too small to accurately measure most tremors. However, we developed an inverse filter to estimate the actual tremor amplitude and phase despite the limited bandwidth. Over the combinations of frequency and amplitude mentioned above, the inverse filter estimated the actual tremor amplitude and phase with errors of $3 \%$ and $2 \%$, respectively. 


\section{Introduction}

Tremor is considered the most common movement disorder [1, 2]. It can occur in a variety of movement disorders, including Essential Tremor, Dystonia, Parkinson's disease, and Cerebellar Ataxia. Tremor causes an involuntary shaking that manifests most commonly in the upper limbs, where it can significantly interfere with activities of daily living, resulting in disability [3]. Whereas tremor frequency is usually between 4 and $8 \mathrm{~Hz}$, tremor amplitude varies greatly, ranging from barely perceivable to $10 \mathrm{~cm}$ or more, though most tremors are on the order millimeters to centimeters. ${ }^{1}$

Despite its prevalence and life-changing impact on many patients, tremor is still diagnosed clinically using subjective, qualitative rating scales: clinicians visually observe a patient's hand tremor under different conditions (e.g. at rest, holding a posture, and during movement) and judge the amplitude or severity of the tremor on a numerical rating scale (e.g. 0-4) [5-8]. Such assessments lack the resolution, accuracy, and repeatability needed to make early adjustments to interventions (medication or deep brain stimulation), determine other tremor characteristics such as frequency or regularity with any reasonable degree of accuracy, track subtle changes over time, or improve the ability to differentiate between tremor disorders.

Markerless motion capture (MMC) provides a potential solution. Although conventional motion capture systems, inertial measurement units, or robotic interfaces can be used to accurately measure tremor, they are prohibitively time-intensive and/or expensive for routine clinical use. For a high-resolution tremor assessment to be adopted in clinical settings, it must be quick and lowcost. Recent advances in markerless motion capture (MMC) driven by the gaming industry have created MMC systems that require virtually no setup time, are low-cost, and are approaching the accuracy needed to assess movement impairments. For example, the Leap Motion Controller (Leap Motion, San Francisco, CA), which costs approximately US\$80, tracks fingers and hands with a sampling rate around 100 samples per second, and with static and dynamic accuracy around 0.5 $\mathrm{mm}$ and $1.2 \mathrm{~mm}$, respectively $[9,10]$.

The setup time, cost, and accuracy of the Leap Motion Controller (LMC) make it a potential tool for widespread clinical assessment of tremor, but its frequency response (ability to track oscillatory movement such as tremor) has not been characterized. Past characterizations of the LMC have focused on its static and dynamic accuracy. Weichert et al measured the ability of the LMC to track a pointer-like tool attached to the end-effector of an industrial robot [9]. They found a static accuracy of $<0.2 \mathrm{~mm}$ and a dynamic accuracy of $<1.2 \mathrm{~mm}$. Similarly, Guna et al tracked a stationary mannequin hand simultaneously with the LMC and an optoelectronic system and determined the static accuracy to be $<0.5 \mathrm{~mm}$ [10]. However, neither the static nor dynamic accuracy characterize the LMC's frequency response, i.e. its ability to accurately estimate the amplitude and phase of oscillatory movements. Therefore, it is unknown if the LMC has the

\footnotetext{
${ }^{1}$ For example, on a scale of 0 to 3 (0: no visible tremor; 1: low amplitude/barely perceivable; 2 : amplitude of 1-2 $\mathrm{cm}$; 3: amplitude $>2 \mathrm{~cm}$ ), the mean score among 831 subjects with Essential Tremor was $1 \pm 0.7(\mathrm{mean} \pm \mathrm{SD})$ during maintenance of a posture and $1.8 \pm 0.6$ during movement [4].
} 
bandwidth required to measure tremor. The purpose of this study was to characterize the frequency response of the LMC and determine its suitability to measure tremor.

\section{Methods}

\section{Experimental Setup}

A stiff mannequin hand was rigidly attached to a linear oscillator (Rectilinear Plant system by Educational Control Products, Bell Canyon, CA) consisting of a sled driven by a motor along a linear bearing (Figure 1). The system also included a CP-850-HHC High Resolution Encoder (Computer Optical Products, Inc., Chatsworth, California), referred to hereafter as HR Encoder, that recorded the position of the sled at 160 counts per mm with an accuracy of \pm 12 arc seconds. This system was controlled using a custom LabView (National Instruments, Austin, Texas) program on a desktop computer running Windows 7 operating system with an Intel ${ }^{\circledR}$ Core ${ }^{\text {TM }} 2$ Duo E8500 processor, 4 GB of RAM, and an NVIDIA Quadro NVS 290 graphics card. In addition, we measured the position of the mannequin hand using a Leap Motion Controller (LMC) that was mounted to a vertical surface in such a way that its cameras were oriented horizontally and the hand was centered in its field of view (Figure 1). At the near and far limits of the linear bearing, the distance from the LMC to the hand was $130 \mathrm{~mm}$ and $200 \mathrm{~mm}$, respectively. The custom LabView program simultaneously recorded (at approximately $100 \mathrm{samples} / \mathrm{sec}$ ) the position of the sled measured by the HR encoder and the 3-dimensional position of all the fingertips and the palm of the mannequin hand measured by the LMC running software version $2.3 .1+31549$.

Immediately before the experiment, the LMC was calibrated using its calibration software, resulting in a score of 95 out of 100. Additionally, we conducted a trial run to measure the LMC's hand confidence value (HCV), a parameter between 0 and 1 that indicates how well the internal hand model of the LMC fits the observed data points. We measured the HCV to be 1, indicating a clearly discernable hand.

\section{Experimental Protocol}

We conducted 107 trials where the mannequin hand was driven in a sinusoidal manner at a variety of frequencies and amplitudes for 40 seconds. During each trial, the frequency and amplitude were kept constant at a unique frequency-amplitude combination. Frequencies ranged between 1 and 15 $\mathrm{Hz}$, and amplitudes between 0.08 and $28.3 \mathrm{~mm}$ (as measured by the HR encoder), with 6-10 trials of different amplitudes at each frequency (Figure 2A).

\section{Data Processing}

The position data from the HR encoder and LMC were interpolated at $100 \mathrm{~Hz}$ with a Piecewise Cubic Hermite Interpolating Polynomial (PCHIP) using Matlab's interp1 function. To characterize the frequency response of the LMC, we treated the LMC as an input-output system (Figure 2B). The input represented the actual displacement of the mannequin hand, assumed to be $A_{\text {in }} \sin \left(2 \pi f t+\phi_{\text {in }}\right)$, where $A_{\text {in }}, f$, and $\phi_{\text {in }}$ were the amplitude, frequency, and phase of the position data measured by the HR encoder. The output represented the measurement of the LMC and was defined as $A_{\text {out }} \sin \left(2 \pi f t+\phi_{\text {out }}\right)$, where $A_{\text {out }}$ and $\phi_{\text {out }}$ were the amplitude and phase measured by the LMC. Following standard system dynamics theory [11], the frequency response 
of the LMC was characterized in terms of the magnitude ratio $M$ and phase shift $\phi$, defined as $M=A_{\text {out }} / A_{\text {in }}$ and $\phi=\phi_{\text {out }}-\phi_{\text {in }}$. We calculated separate values of $M$ and $\phi$ for each of the 107 trials, resulting in discrete values of $M$ and $\phi$ at different values of frequency and actual (input) amplitude.

To determine the values of $f, A_{\text {in }}, \phi_{\text {in }}, A_{\text {out }}$, and $\phi_{\text {out }}$ needed to calculate $M$ and $\phi$, we transformed the data measured by the HR encoder and LMC into the frequency domain via the Fast Fourier Transform (using Matlab's fft function). The resolution in frequency was 1/(40 sec) $=0.025 \mathrm{~Hz}$. The magnitude vs. frequency plot of the $\mathrm{HR}$ encoder data always contained a clearly identifiable peak, so we took the frequency and magnitude of this peak to be $f$ and $A_{\text {in }}$. The input phase, $\phi_{\text {in }}$, was determined as the phase of the HR encoder data at $f$. Likewise, $A_{\text {out }}$ and $\phi_{\text {out }}$ were determined as the magnitude and phase of the LMC data at $f$.

\section{Data Analysis}

\section{Frequency response as a function of input amplitude}

Since the magnitude ratio and phase shift of a linear system are independent of input amplitude, linear systems are characterized by a single frequency response (magnitude ratio vs. frequency and phase vs. frequency) no matter the input amplitude. We found the magnitude ratio and phase shift of the LMC to depend on input amplitude (see Results), indicating a nonlinear input-output relationship necessitating separate frequency response characterizations for different input amplitudes. However, the input amplitudes were not consistent across input frequencies (Figure $2 \mathrm{~A}$ ), so to determine the frequency response for different input amplitudes, we grouped the input amplitudes into 6 bins $(0-0.5 \mathrm{~mm}, 0.5-1.2 \mathrm{~mm}, 1.2-3 \mathrm{~mm}, 3-5 \mathrm{~mm}, 5-10 \mathrm{~mm}$, and $10-30 \mathrm{~mm})$ and calculated the average magnitude ratio and phase shift in each bin at each frequency $(1-15 \mathrm{~Hz})$.

\section{Bandwidth}

We found that the magnitude ratio of the LMC decreased with increasing frequency (see Results), indicating that the LMC acts as a low-pass filter. The bandwidth of a system represents the band of input frequencies that pass relatively unperturbed through the system, defined as the band of frequencies for which $M \geq 0.707$. Because of the nonlinear input-output relationship of the LMC, the bandwidth of the LMC varies with amplitude. The limited range of input amplitudes and frequencies (Figure 2A) did not allow an exact determination of bandwidth for all input amplitudes. However, we obtained a rough estimate of the bandwidth of each amplitude bin by fitting the magnitude ratios of second-order and third-order linear systems to the data (separately for each amplitude bin) and determining the frequency at which the magnitude ratio crossed 0.707. Rounded to the nearest integer, the resulting bandwidths were the same for the second-order and third-order systems.

\section{Inverse Filter}

Because of the limited frequency response of the LMC, the amplitude and phase measured (output) by the LMC are not, in general, equal to the actual (input) amplitude and phase, i.e. $A_{\text {out }} \neq A_{\text {in }}$ and $\phi_{\text {out }} \neq \phi_{\text {in }}$ (because $M \neq 1$ and $\phi \neq 0$ ). However, if the variation of $M$ and $\phi$ were known as continuous functions of the frequency and measured (output) amplitude, i.e. $M=g\left(f, A_{\text {out }}\right)$ and $\phi=h\left(f, A_{\text {out }}\right)$, the actual (input) amplitude and phase could be estimated from measured 
(output) values of amplitude and phase: $A_{\text {in }}=A_{\text {out }} / g\left(f, A_{\text {out }}\right)$ and $\phi_{\text {in }}=\phi_{\text {out }}-h\left(f, A_{\text {out }}\right)$. We derived $g$ as a two-dimensional polynomial regression of $M$ over $f$ and $A_{\text {out }}$, and likewise $h$ as a two-dimensional polynomial regression of $\phi$ over $f$ and $A_{\text {out }}$ :

Equation 1

$$
M=g\left(f, A_{\text {out }}\right)=\sum_{i=0}^{N} \sum_{j=0}^{N} a_{i j} f^{i} A_{\text {out }}{ }^{j}
$$

Equation 2

$$
\phi=h\left(f, A_{\text {out }}\right)=\sum_{i=0}^{N} \sum_{j=0}^{N} b_{i j} f^{i} A_{\text {out }}{ }^{j}
$$

where $a_{i j}$ and $b_{i j}$ are coefficients and $N$ is the order of the polynomial regression. To determine the optimal order, we repeated the regression for polynomial orders between 1 and 9 ( $N=$ $1,2, \ldots, 9)$ and evaluated for each order the error, defined as the mean absolute difference between the values of $A_{\text {in }}$ and $\phi_{\text {in }}$ measured directly by the HR encoder and those estimated as $A_{\text {in }}=$ $A_{\text {out }} / g\left(f, A_{\text {out }}\right)$ and $\phi_{\text {in }}=\phi_{\text {out }}-h\left(f, A_{\text {out }}\right)$ at the corresponding frequency and amplitude. In calculating the error, we excluded trials with actual (input) amplitude below the dynamic accuracy of the LMC (1.2mm).

\section{Results}

The amplitude and phase of the input were clearly discernable in the frequency domain for all amplitudes and frequencies (Figure 3). Likewise, the amplitude and phase of the output were clearly discernable in the frequency domain, but only when the amplitude of the input was above the dynamic accuracy $(1.2 \mathrm{~mm})$. Although the motor was always commanded to drive at a single input frequency, the resulting response of the linear oscillator system sometimes exhibited significant oscillations at multiple frequencies (e.g., see position plot in second-to-last row of Figure 3). Nevertheless, for all trials we focused only on the amplitudes and phases of the input and output at the commanded frequency. As clearly seen in Figure 3, the relationship between input amplitude and output amplitude (and between input phase and output phase) depended not only on frequency, but also on input amplitude, highlighting the nonlinear nature of the LMC.

The magnitude ratio generally decreased with increasing frequency and decreasing amplitude, but less so for the palm than for the fingers (see Figure S1 in Supplementary Material). At higher frequencies $(\geq 8 \mathrm{~Hz})$, the magnitude ratios of all fingers were at least an order of magnitude smaller than that of the palm. Therefore, for the remainder of the analysis, we focused on the position of the palm.

\section{Frequency response as a function of input amplitude}

The magnitude ratio of palm position ranged from 0.99 to 0.0001 (Figure 4A). For input amplitudes above the dynamic accuracy of the LMC $(1.2 \mathrm{~mm})$, the magnitude ratio clearly decreased with increasing frequency and decreasing amplitude. For input amplitudes below the 
dynamic accuracy, the magnitude ratio remained low $(<0.3)$ independent of frequency and did not exhibit a clear pattern with increasing frequency.

For input amplitudes above the dynamic accuracy, the phase shift showed a clear, nearly linear increase (in the negative direction) with increasing frequency (Figure 4B). For input amplitudes below the dynamic accuracy, the phase shift was $-120^{\circ}$ or more negative, and became increasingly negative with increasing frequency, though not monotonically.

\section{Bandwidth}

We found rough estimates of the bandwidth for bins $10-30 \mathrm{~mm}, 5-10 \mathrm{~mm}, 3-5 \mathrm{~mm}$, and $1.2-3 \mathrm{~mm}$ to be $5 \mathrm{~Hz}, 5 \mathrm{~Hz}, 4 \mathrm{~Hz}$, and $3 \mathrm{~Hz}$, respectively. The two smallest bins $(0.5-1.2 \mathrm{~mm}$, and $0-0.5 \mathrm{~mm})$ were not considered since these bins are below the dynamic accuracy of the LMC.

\section{Inverse Filter}

We estimated the magnitude ratio and phase shift as continuous functions of frequency and output amplitude: $M=g\left(f, A_{\text {out }}\right)$ and $\phi=h\left(f, A_{\text {out }}\right)$. The accuracy of these fit functions depended on their polynomial order (Figure 5). Applied to the experimental data, average errors in estimating the actual magnitude ratio and phase shift ranged from 20 and 10\% (for a first-order polynomial fit) to 0.5 and $0.25 \%$ (for a ninth-order fit), respectively. The average error plateaued somewhat around fifth-order fits, with errors in magnitude ratio and phase shift at $3.3 \pm 4.3 \%$ and $2.3 \pm 2.2 \%$, respectively. We selected the fifth-order fit because it provides a balance between accuracy and tractability, and we present regression coefficients $a_{i j}$ and $b_{i j}$ in Table 1 and Table 2.

Functions $g$ and $h$ behave as expected within the range of amplitudes and frequencies over which they were interpolated; the magnitude ratio decreases and the phase shift increases (becomes more negative) with increasing frequency and decreasing input amplitude. Within this region, the fit between the measured data and functions $g$ and $h$ is good (around $3 \%$ for $M$ and $2 \%$ for $\phi$, as mentioned above), and functions $g$ and $h$ can be used as inverse filters (Figure 2B) to estimate the actual (input) amplitude and phase from the measured (output) amplitude and phase: $A_{\text {in }}=$ $A_{\text {out }} / g\left(f, A_{\text {out }}\right)$ and $\phi_{\text {in }}=\phi_{\text {out }}-h\left(f, A_{\text {out }}\right)$. However, the fit rapidly deteriorates outside of this region, resulting in unexpected behavior (see Figure S2 in Supplementary Material). Therefore, functions $g$ and $h$ cannot be used reliably to extrapolate beyond the region over which they were regressed.

\section{Discussion}

Currently, clinicians assess a patient's tremor using subjective clinical rating scales. Markerless motion capture (MMC) systems provide a potential solution; they are low-cost, require no setup time between patients, and are beginning to have sufficient accuracy for clinical use. As the Leap Motion Controller (LMC) is an MMC system specifically designed to track hand motion, it may be particularly well-suited to track tremor in clinical settings. However, even though prior studies have characterized its static and dynamic accuracy $[9,10]$, its ability to track tremor has not been characterized. 


\section{Frequency Response}

\section{Amplitude}

The LMC acts as a low-pass filter in that the magnitude ratio decreases and the phase shift increases (becomes more negative) with increasing frequency. This filtering may be due to limitations in the hardware or the signal processing software of the LMC. Given that most cameras have high bandwidth, it is likely that the bandwidth we observed is imposed by the proprietary signal processing software of the LMC.

We found the bandwidth of the LMC to be around $5 \mathrm{~Hz}$ or less, depending on the tremor amplitude. Since most tremors have frequencies between 4 and 12Hz [3], without additional processing (see below) the LMC cannot be used to reliably measure the amplitude of most tremors, although it could still be used to determine frequency and regularity.

\section{Phase Shift}

A non-zero phase shift has little adverse effect on measurements of tremor, including amplitude, frequency, and regularity. However, phase shift can be detrimental in the real-time display of oscillatory movements (e.g. finger tapping) because the phase of the movement will not match the phase displayed on a graphical user interface (GUI). This becomes especially problematic when the GUI is involved in a feedback loop, such as when the user is using the GUI to control his/her oscillatory movements.

\section{Inverse Filter}

The inverse filter provides a simple method for estimating the actual displacement and phase from the displacement and phase measured by the LMC (Figure 2B). In the end we chose a fifth-order fit because it yielded an acceptable balance of accuracy and tractability.

\section{Frequency response of other tremor-measuring systems}

As far as we are aware, the only previous investigation of frequency response of an MMC system was performed by Kvalbein on the Microsoft Kinect system [12]. Kvalbein found the Kinect system to have a bandwidth of approximately $2 \mathrm{~Hz}$ for oscillation amplitudes of 10-20 mm. In comparison, the bandwidth of the LMC for oscillation amplitudes of 10-30 mm was $5 \mathrm{~Hz}$. In addition, the accuracy of the Kinect system is lower than that of the LMC. Whereas the static accuracy of the Kinect system is 5-50 $\mathrm{mm}$ (depending on where the object is located in the sensing volume), the static and dynamic accuracy of the LMC are $0.2-0.5 \mathrm{~mm}$ and $1.2 \mathrm{~mm}$, respectively [9, 10]. For these reasons, the LMC is better suited to track tremor of the hands.

When tremor is measured with a sensor (usually in a research setting), it is most commonly measured with accelerometers [13-15]. Though less common, tremor is also sometimes measured using gyroscopes $[13,14,16]$ or optoelectronic position sensors (i.e. motion capture systems with markers) [17, 18]. Accelerometers, gyroscopes, and optoelectronic sensors typically have bandwidths that are much larger (often by multiple orders of magnitude) than the bandwidth of tremor $[19,20]$. However, they require the application of sensors or markers and often skilled postprocessing, which can be prohibitively time-intensive for clinical settings. 


\section{Limitations}

Tremor is a complex kinematic phenomenon including both rotation and translation. To clarify, tremorogenic muscle activity produces tremorogenic torque in one or multiple joints, which results in rotational (i.e. angular) displacement at these and other joints [21]; in a linkage such as the upper limb, these rotational displacements sum, producing both rotational and translational displacements of the end effector (hands/fingers). However, the linkage tends to amplify the translational displacements of the end effector (relative to the rotational displacements), so tremor is usually observed/recorded and expressed as translational displacement (or velocity or acceleration) of the hands/fingers, ignoring rotational displacements. Similarly, our study focused on the translational displacements of the hand/fingers (even though the LMC measures the rotational displacements of the hand/fingers as well). Furthermore, we investigated the ability of the LMC to track tremor in only a single direction (the y-direction - see Figure 1) even though tremor generally involves three-dimensional movement. According to Weichert et al, the accuracy in the z-direction is similar to that of the y-direction, and the accuracy in the x-direction is better than in the y-or z-direction [22]. We suspect the bandwidths in the different directions to follow this same trend, i.e. we suspect the bandwidth in the z-direction to be similar to that of the ydirection, and the bandwidth in the x-direction to be better than the bandwidth in the $y$ - and $\mathrm{z}$ directions.

Because of limitations in the linear oscillator system driving the mannequin hand, we were unable to generate large amplitudes at high frequencies. Consequently, the characterization presented here is only valid for small oscillation amplitudes when the oscillation frequency is high (see Figure 2A). Also, we commanded the frequency of the oscillations, not the amplitude. Calculating the frequency response (magnitude ratio and phase shift as functions of frequency) for a given input amplitude therefore required us to average responses across a range of frequencies within a group, reducing the accuracy of the bandwidth estimates.

The errors associated with the inverse filter $3.3 \%$ and $2.3 \%$ for estimating the actual magnitude ratio and phase shift, respectively) were calculated on the same data used to create the inverse filter. We'd expect the errors associated with the estimated magnitude ratio and phase shift calculated from new data to be higher. Also, as noted above, the accuracy of the inverse filter rapidly declines outside of the range spanned by the data used to create the filter, so the equations cannot be used reliably outside of this range (see Figure S2 in Supplementary Material).

We were careful to maximize the accuracy of the LMC, resulting in high calibration scores ( $\geq 95 / 100$ for all tests). Thus, the data presented in this paper can be considered a best-case scenario. Due to constraints on time and space as well as limited experience with the LMC, clinical settings may not allow such precautions and could result in greater measurement errors than those presented here.

\section{Conclusion}

We conclude that the LMC can accurately measure low-frequency, high-amplitude tremor with reasonable accuracy. In contrast, the LMC is unable to accurately measure tremor at high frequency or low amplitude without additional processing. However, using the inverse filter 
presented here, it is possible to estimate the actual tremor amplitude and phase from the measured amplitude and phase (for tremor amplitudes above the sensor's dynamic accuracy of $1.2 \mathrm{~mm}$ ). As gaming technology drives the further development of MMC systems, we expect their ability to accurately measure tremor to further increase. 


\section{References}

[1] E. D. Louis and J. J. Ferreira, "How Common Is the Most Common Adult Movement Disorder? Update on the Worldwide Prevalence of Essential Tremor," Movement Disorders, vol. 25, pp. 534-541, Apr 2010.

[2] E. D. Louis and R. Ottman, "How many people in the USA have essential tremor? Deriving a population estimate based on epidemiological data," Tremor Other Hyperkinet Mov, vol. 4, p. 259, 2014.

[3] K. P. Bhatia, P. Bain, N. Bajaj, R. J. Elble, M. Hallett, E. D. Louis, et al., "Consensus Statement on the classification of tremors. from the task force on tremor of the International Parkinson and Movement Disorder Society," Mov Disord, vol. 33, pp. 75-87, Jan 2018.

[4] E. D. Louis, N. Hernandez, and M. Michalec, "Prevalence and correlates of rest tremor in essential tremor: cross-sectional survey of 831 patients across four distinct cohorts," European Journal of Neurology, vol. 22, pp. 927-932, Jun 2015.

[5] C. G. Goetz, S. Fahn, P. Matrinez-Martin, W. Poewe, C. Sampaio, G. T. Stebbins, et al., "Movement disorder society-sponsored revision of the Unified Parkinson's Disease Rating Scale (MDS-UPDRS): Process, format, and clinimetric testing plan," Movement Disorders, vol. 22, pp. 41-47, 2007.

[6] P. Trouillas, et al., "International Cooperative Ataxia Rating Scale for pharmacological assessment of the cerebellar syndrome," Journal of the Neurological Sciences, vol. 145, pp. 205-211, 1997.

[7] R. Elble, C. Comella, S. Fahn, M. Hallett, J. Jankovic, J. Juncos, et al., "The essential tremor rating assessment scale (TETRAS)," Movement Disorders, vol. 23, pp. S357-S357, 2008.

[8] S. Fahn, et al., "Clinical Rating Scale for Tremor," in Parkinson's Disease and Movement Disorders, ed Baltimore-Munich: Urban \& Schwarzenberg, 1988, pp. 225-234.

[9] F. Weichert, D. Bachmann, B. Rudak, and D. Fisseler, "Analysis of the accuracy and robustness of the leap motion controller," Sensors (Basel), vol. 13, pp. 6380-93, May 14 2013.

[10] J. Guna, G. Jakus, M. Pogacnik, S. Tomazic, and J. Sodnik, "An analysis of the precision and reliability of the leap motion sensor and its suitability for static and dynamic tracking," Sensors (Basel), vol. 14, pp. 3702-20, Feb 212014.

[11] W. Palm, System Dynamics. New York, NY: McGraw Hill, 2014.

[12] M. Kvalbein, "The use of a 3D sensor (Kinect) for robot motion compensation," 2012.

[13] R. J. Elble and J. McNames, "Using Portable Transducers to Measure Tremor Severity," Tremor Other Hyperkinet Mov (N Y), vol. 6, p. 375, 2016.

[14] G. Grimaldi and M. Manto, "Neurological Tremor: Sensors, Signal Processing and Emerging Applications," Sensors, vol. 10, pp. 1399-1422, Feb 2010.

[15] B. K. Scanlon, B. E. Levin, D. A. Nation, H. L. Katzen, A. Guevara-Salcedo, C. Singer, et al., "An accelerometry-based study of lower and upper limb tremor in Parkinson's disease," Journal of Clinical Neuroscience, vol. 20, pp. 827-830, Jun 2013.

[16] J. A. Gallego, E. Rocon, J. O. Roa, J. C. Moreno, and J. L. Pons, "Real-Time Estimation of Pathological Tremor Parameters from Gyroscope Data," Sensors, vol. 10, pp. 21292149, Mar 2010.

[17] G. Deuschl, R. Wenzelburger, K. Loffler, J. Raethjen, and H. Stolze, "Essential tremor and cerebellar dysfunction clinical and kinematic analysis of intention tremor," Brain, vol. 123 ( Pt 8), pp. 1568-80, Aug 2000. 
[18] R. A. MacLachlan and C. N. Riviere, "High-Speed Microscale Optical Tracking Using Digital Frequency-Domain Multiplexing," Ieee Transactions on Instrumentation and Measurement, vol. 58, pp. 1991-2001, Jun 2009.

[19] S. Beeby, MEMS mechanical sensors. Boston: Artech House, 2004.

[20] J. r. Haus, Optical sensors : basics and applications. Weinheim: Wiley-VCH, 2010.

[21] A. D. Davidson and S. K. Charles, "Fundamental Principles of Tremor Propagation in the Upper Limb," Annals of Biomedical Engineering, vol. 45, pp. 1133-1147, 2017.

[22] F. Weichert, D. Bachmann, B. Rudak, and D. Fisseler, "Analysis of the Accuracy and Robustness of the Leap Motion Controller," Sensors, vol. 13, pp. 6380-6393, May 2013. 
Table 1: Magnitude ratio coefficients $a_{i j}$ for Equation 1

\section{Magnitude Ratio Coefficients}

\begin{tabular}{|c|l|l|l|l|l|l|}
\hline $\mathbf{i} / \mathbf{j}$ & \multicolumn{1}{|c|}{$\mathbf{0}$} & \multicolumn{1}{c|}{$\mathbf{1}$} & \multicolumn{1}{c|}{$\mathbf{2}$} & \multicolumn{1}{c|}{$\mathbf{3}$} & \multicolumn{1}{c|}{$\mathbf{4}$} & \multicolumn{1}{c|}{$\mathbf{5}$} \\
\hline $\mathbf{0}$ & $1.65 \times 10^{-1}$ & $2.36 \times 10^{-1}$ & $-2.14 \times 10^{-2}$ & $7.85 \times 10^{-4}$ & $-1.61 \times 10^{-5}$ & $3.33 \times 10^{-7}$ \\
\hline $\mathbf{1}$ & $3.84 \times 10^{-1}$ & $-1.12 \times 10^{-1}$ & $5.43 \times 10^{-3}$ & $2.67 \times 10^{-4}$ & $-1.55 \times 10^{-5}$ & 0 \\
\hline $\mathbf{2}$ & $-1.92 \times 10^{-1}$ & $6.18 \times 10^{-2}$ & $-5.14 \times 10^{-3}$ & $1.11 \times 10^{-4}$ & 0 & 0 \\
\hline $\mathbf{3}$ & $3.37 \times 10^{-2}$ & $-9.20 \times 10^{-3}$ & $4.63 \times 10^{-4}$ & 0 & 0 & 0 \\
\hline $\mathbf{4}$ & $-2.50 \times 10^{-3}$ & $4.14 \times 10^{-4}$ & 0 & 0 & 0 & 0 \\
\hline $\mathbf{5}$ & $6.63 \times 10^{-5}$ & 0 & 0 & 0 & 0 & 0 \\
\hline
\end{tabular}

Table 2: Phase shift coefficients $b_{i j}$ for Equation 2

\section{Phase Shift Coefficients}

\begin{tabular}{|c|l|l|l|l|l|l|}
\hline $\mathbf{i} / \mathbf{j}$ & \multicolumn{1}{|c|}{$\mathbf{0}$} & \multicolumn{1}{c|}{$\mathbf{1}$} & \multicolumn{1}{c|}{$\mathbf{2}$} & \multicolumn{1}{c|}{$\mathbf{3}$} & \multicolumn{1}{c|}{$\mathbf{4}$} & \multicolumn{1}{c|}{$\mathbf{5}$} \\
\hline $\mathbf{0}$ & $-1.11 \times 10^{2}$ & $3.69 \times 10^{1}$ & $-4.22 \times 10^{0}$ & $1.99 \times 10^{-1}$ & $-.453 \times 10^{-3}$ & $5.49 \times 10^{-5}$ \\
\hline $\mathbf{1}$ & $4.62 \times 10^{1}$ & $-2.77 \times 10^{1}$ & $2.72 \times 10^{0}$ & $-6.81 \times 10^{-2}$ & $-1.44 \times 10^{-4}$ & 0 \\
\hline $\mathbf{2}$ & $-22.66 \times 10^{1}$ & $1.16 \times 10^{1}$ & $-1.01 \times 10^{0}$ & $2.16 \times 10^{-2}$ & 0 & 0 \\
\hline $\mathbf{3}$ & $4.77 \times 10^{0}$ & $-1.59 \times 10^{0}$ & $8.00 \times 10^{-2}$ & 0 & 0 & 0 \\
\hline $\mathbf{4}$ & $-3.60 \times 10^{-1}$ & $6.72 \times 10^{-2}$ & 0 & 0 & 0 & 0 \\
\hline $\mathbf{5}$ & $9.65 \times 10^{-3}$ & 0 & 0 & 0 & 0 & 0 \\
\hline
\end{tabular}




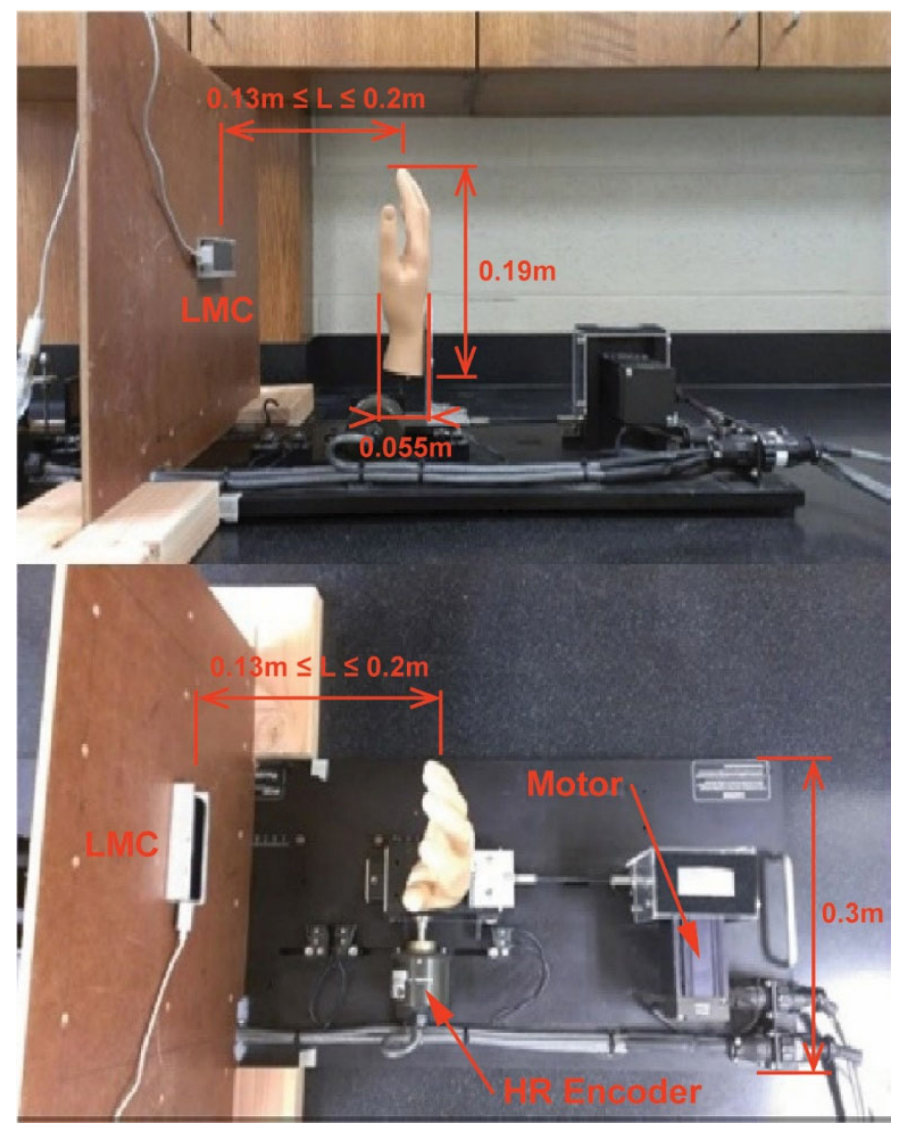

Figure 1: Side and top views of the experimental setup. A mannequin hand was mounted to the sled of a linear oscillating system and moved back and forth in a sinusoidal manner. The position of the mannequin hand was measured simultaneously using the Leap Motion Controller (LMC) and a high-resolution encoder (HR Encoder). 


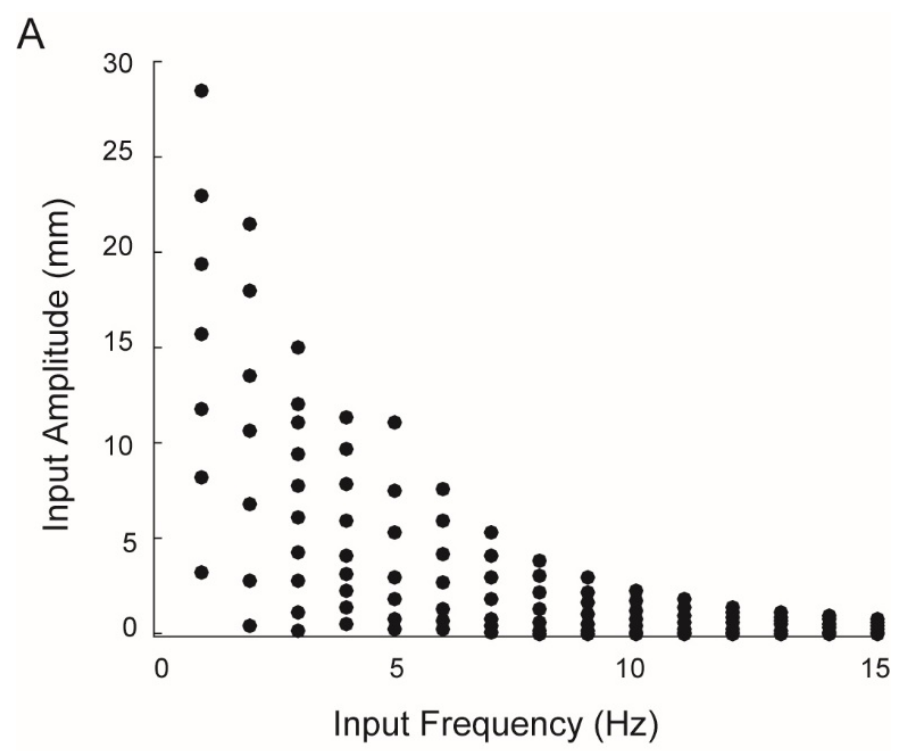

B

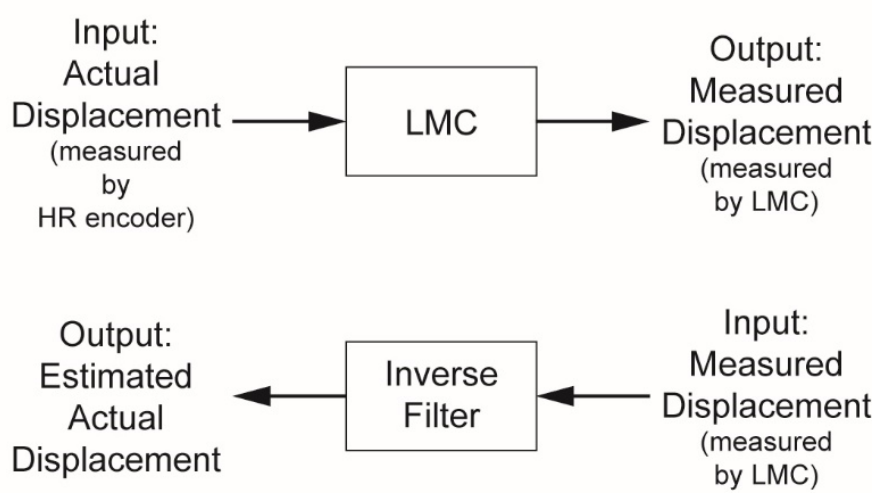

Figure 2. A: The mannequin hand was oscillated back and forth in a sinusoidal manner at the 107 different frequency-amplitude combinations shown here. B: To characterize the frequency response of the LMC, we treated it as an input-output system. Top flow diagram: The input to the LMC was the actual displacement of the mannequin hand, assumed to be the same as the displacement measured by the HR encoder. The output of the LMC was the displacement measured by the LMC. Bottom flow diagram: Characterizing and inverting the frequency response of the $L M C$, we created an inverse filter that can be used to estimate the actual displacement from the displacement measured by the LMC. 
Input

Position vs. Time (mm)

FFT Magnitude (mm)

FFT Phase (degrees)

$1 \mathrm{~Hz}$

$28 \mathrm{~mm}$
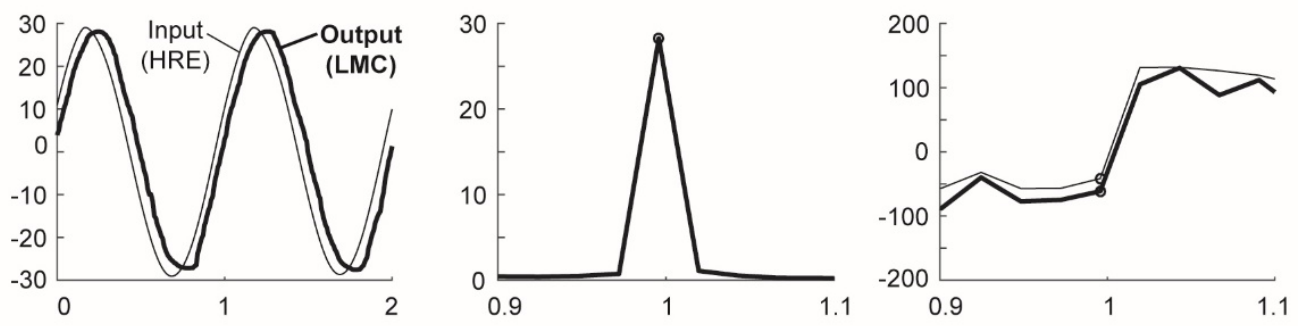

$1 \mathrm{~Hz}$

$5.6 \mathrm{~mm}$
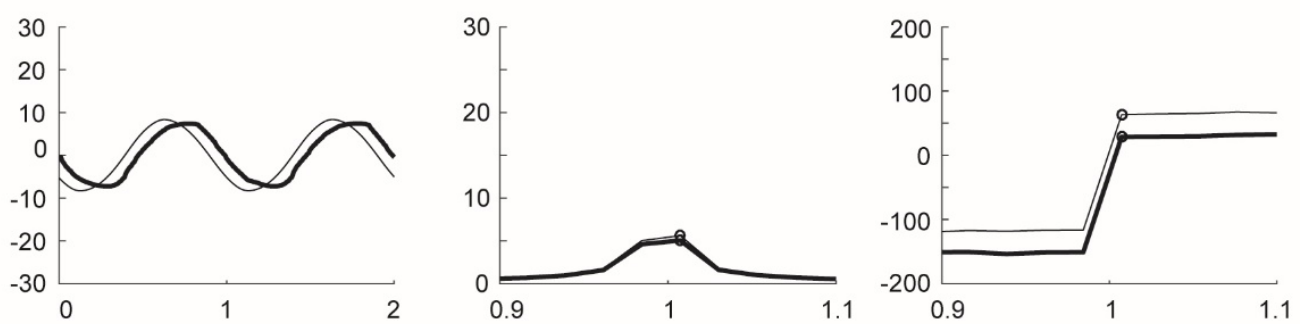

$6 \mathrm{~Hz}$

$6.7 \mathrm{~mm}$
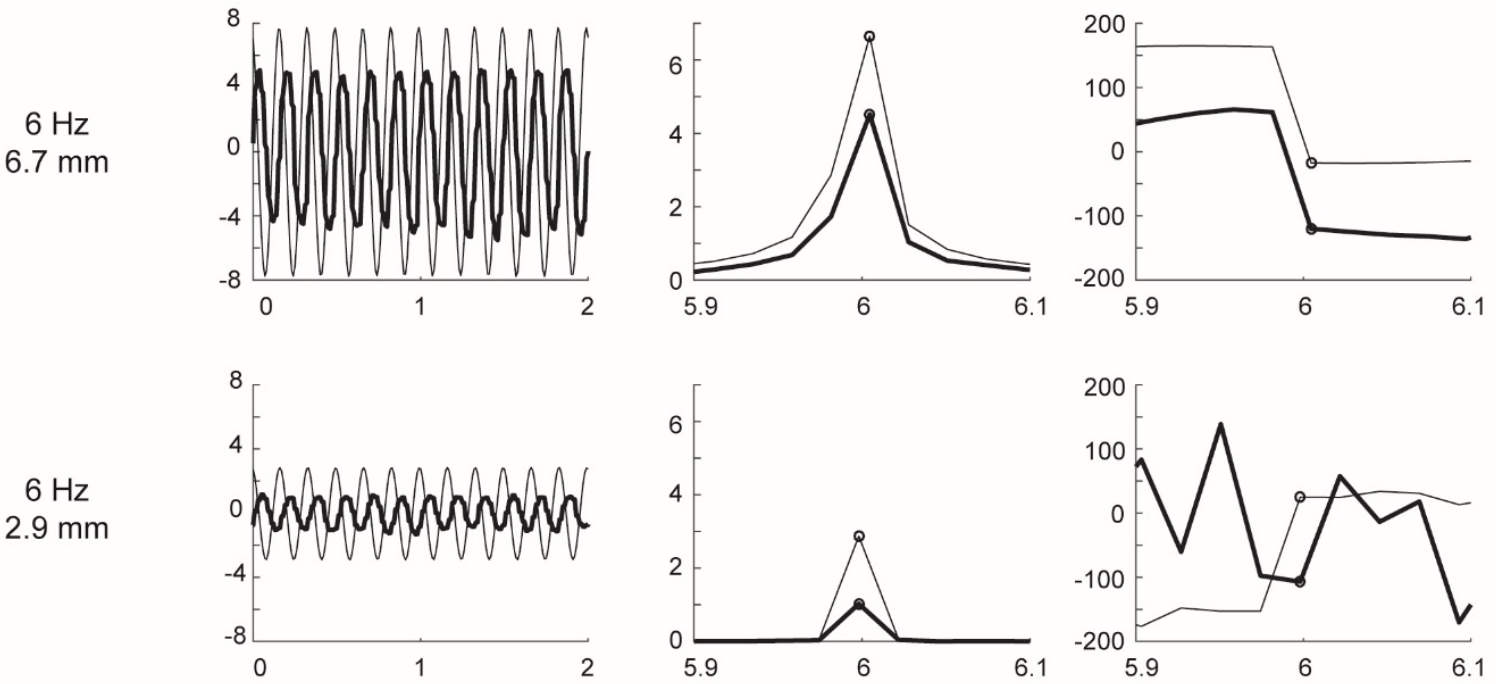

$15 \mathrm{~Hz}$

$0.96 \mathrm{~mm}$
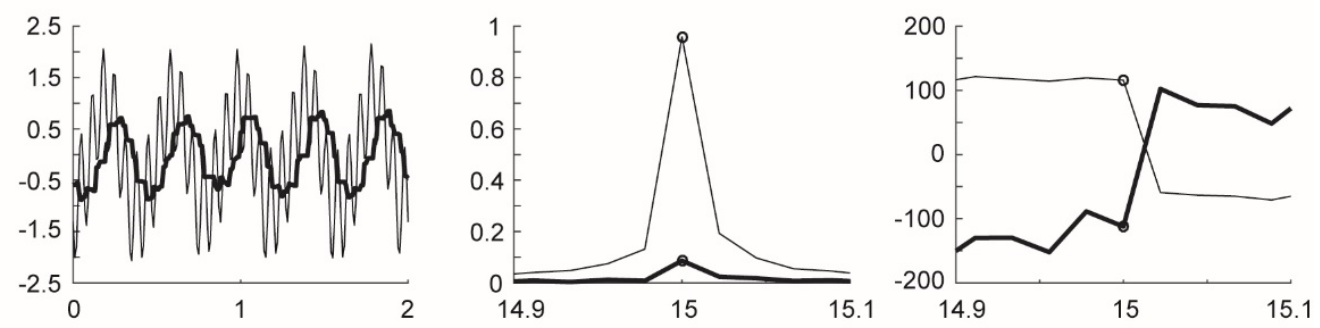

$15 \mathrm{~Hz}$

$0.10 \mathrm{~mm}$
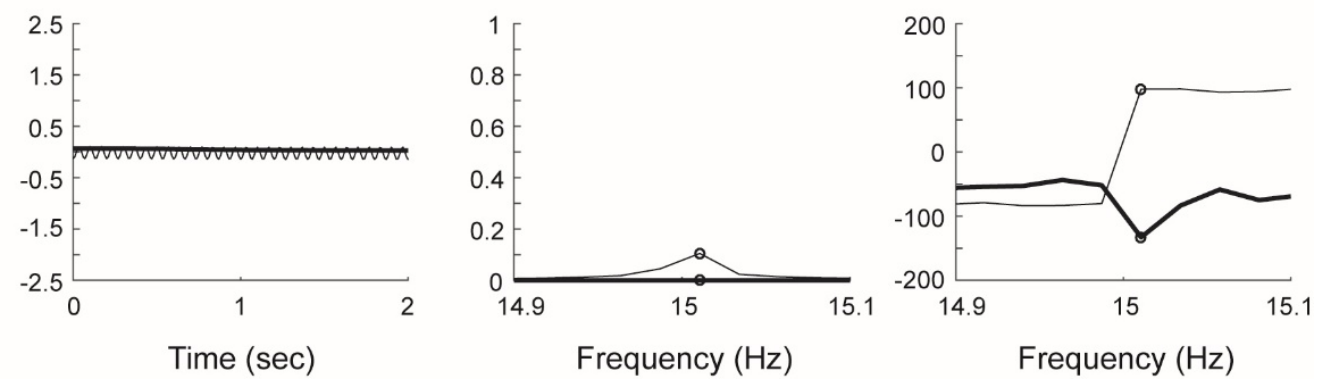
Figure 3. Example data measured by the HR encoder (thin lines) and LMC (thick lines) for the different input frequencies and amplitudes listed in column 1. Shown are position vs. time (column 2) and the magnitude and phase of the data after being transformed by the Fast Fourier Transform (FFT) (columns 3-4). The circles in columns 3-4 indicate the magnitude and phase of the input (displacement measured by the HR encoder) and output (displacement measured by the LMC). These magnitude and phase values were used to calculate the magnitude ratio and phase shift for different input frequency-amplitude combinations. Since the phase shift was calculated as the difference between the input and output phase, the absolute values of the input and output phases are irrelevant. For every input frequency, we included two different input amplitudes (column 1) to show that the relationship between the magnitudes of the input and output and the relationship between the phases of the input and output depend on the input amplitude (and not just the input frequency), indicating that the frequency response of the LMC is nonlinear.
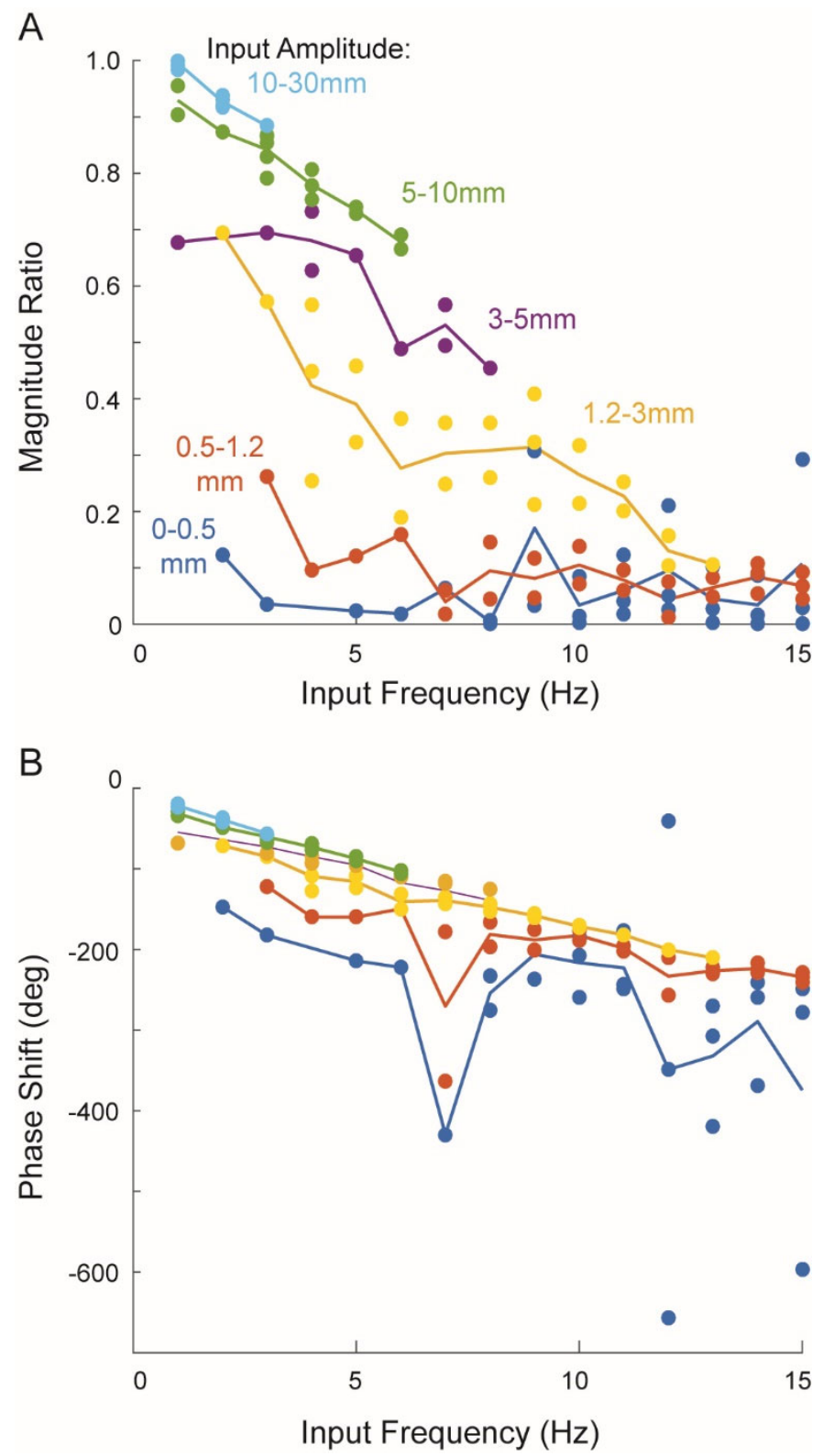

Figure 4. Frequency response plots. Magnitude ratio vs. input frequency (A) and phase shift vs. input frequency (B) for a variety of input amplitudes. The dots represent individual trials, grouped into amplitude groups indicated by different colors. The lines represent the average for each group at a given input frequency. 

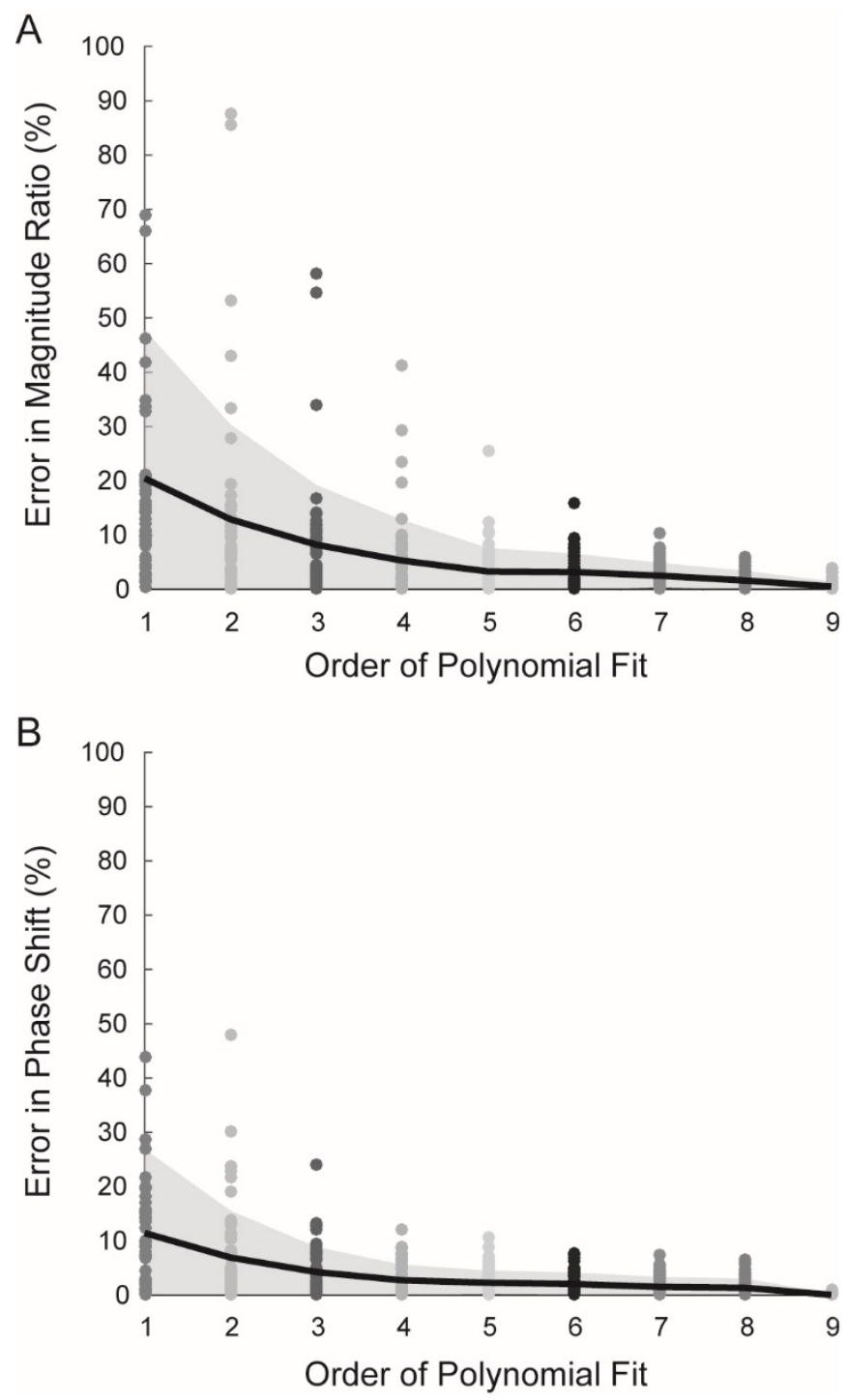

Figure 5. Average percent error in estimating the actual magnitude ratio $(A)$ and phase shift $(B)$ as a function of the order of the polynomial fits (of the experimental data) used for estimation. The gray region represents \pm 1 standard deviation. 


\section{Supplementary Material}

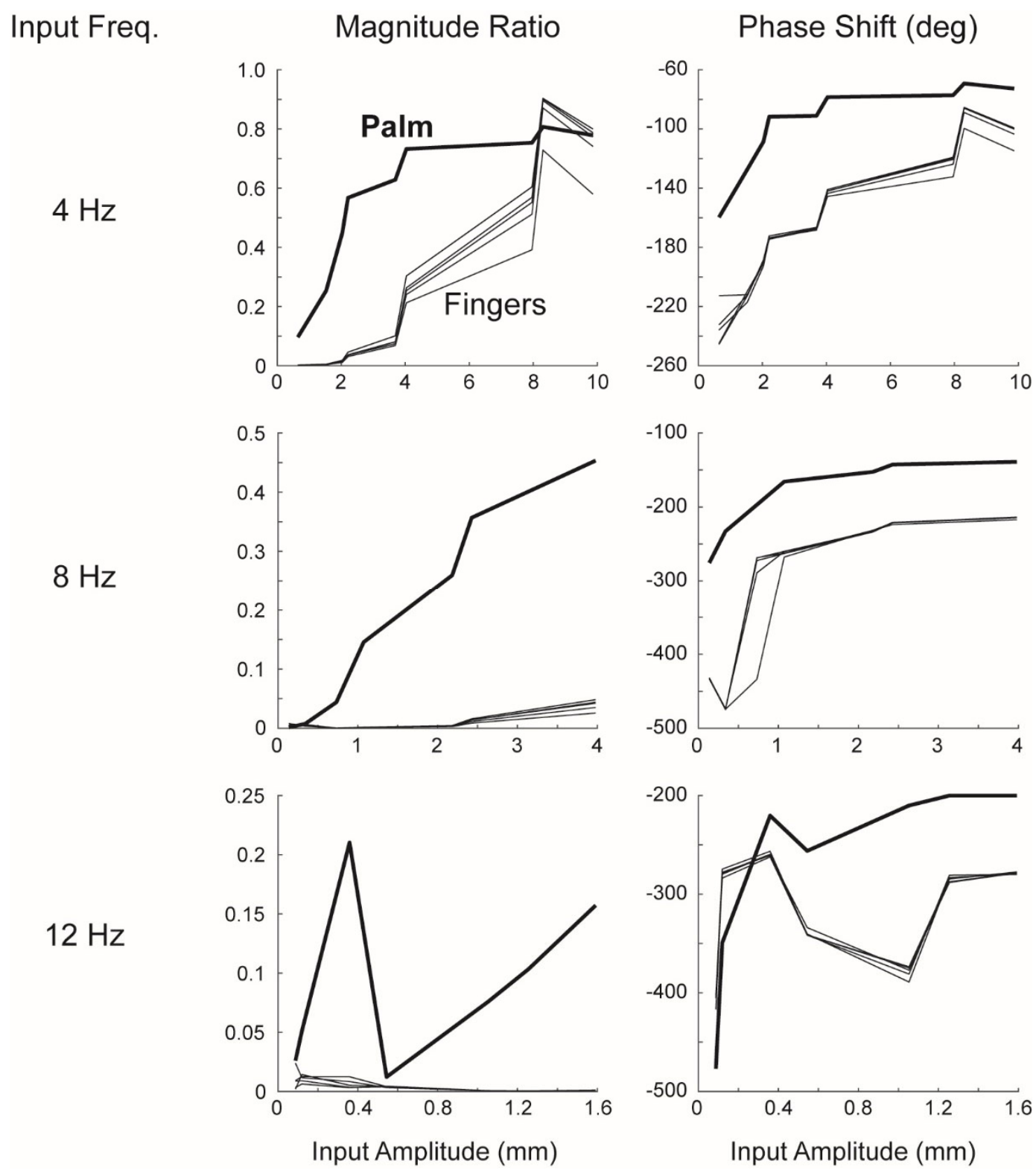

Figure S1: Magnitude ratio and phase shift of the palm (thick lines) and finger tips (thin lines) at three different input frequencies (indicated in the left column) and input amplitudes (indicated on the horizontal axes). The frequency response of the palm was significantly better than the frequency response of the fingers. 

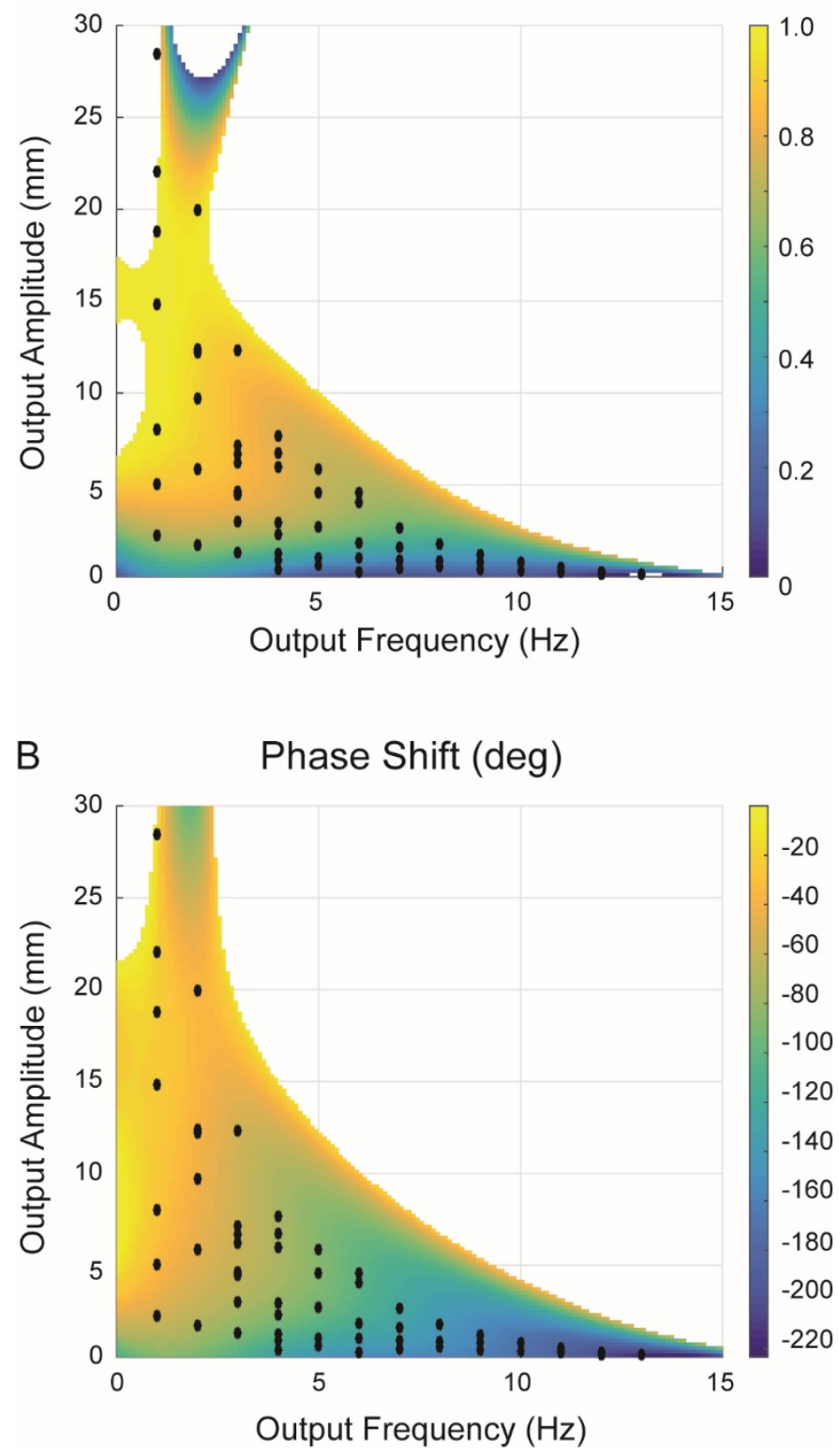

Figure S2: Two-dimensional polynomial fits of the magnitude ratio (A) and phase shift (B) as functions of output amplitude and frequency (the frequency of the input and output are equal). The fits were based on the measurements indicated by the black dots. The accuracy of the fit is high over the region enclosed by the black dots (see Figure 5) but decreases rapidly outside this region, indicating that the fit is unreliable for extrapolation. For example, at an output amplitude of 5mm, the magnitude ratio fit correctly decreases with increasing frequency over the region spanned by the dots; however, for frequencies above approximately $7 \mathrm{~Hz}$, the magnitude ratio fit incorrectly increases. Likewise, for a frequency of $2 \mathrm{~Hz}$, the magnitude ratio fit correctly increases with increasing output amplitude over the region spanned by the dots; however, for amplitudes above $20 \mathrm{~mm}$, the magnitude ratio fit incorrectly decreases. White spaces indicate the regions where the calculated magnitude ratio fell outside the region from 0 to 1 (we did not observe resonance) or the calculated phase shift exceeded $0^{\circ}$. 